\title{
Las Tecnologías de la Informática y la Comunicación (TIC) Integradas a una Unidad Didáctica Interactiva para el Aprendizaje de la Bioquímica y Estructurada a partir del Modelo de Resolución de Problemas
}

Information and Communication Technologies (ICT) Integrated into an Interactive Didactic Unit for Learning Biochemistry Structured from Problem Solving Learning Model

Alfonso Raúl Trujillo Campos, Jesús David Álvarez Roncancio y Arévalo Beltrán Lina Marcela

Proyecto Curricular de Lic. Química. Universidad Distrital Francisco José de Caldas. Bogotá D.C. Colombia. raultujilloc@hotmail.com, davidud1@hotmail.com, asomuvi4@hotmail.com

\section{Resumen}

La didáctica de las ciencias ha desarrollado en los últimos años un importante trabajo en la aplicación de las Tecnologías de la Informática y la Comunicación (TIC) en las aulas. Atendiendo a lo anterior y a la necesidad de adaptar las TIC al contexto educativo y a los avances en el campo de la didáctica y la química, este artículo da a conocer una propuesta que integra estos elementos conjugando la Bioquímica y una Unidad Didáctica Interactiva teniendo en cuenta los planteamientos del modelo de Aprendizaje por Resolución de Problemas.

Para la ejecución del proyecto se tuvo en cuenta los estándares educativos nacionales en Colombia y diversos documentos relacionados con la normatividad curricular concemientes a los conceptos de Bioquímica; así como sus procesos histórico-epistemológicos.

\section{Palabras claves}

Unidad Didáctica, virtualidad, interactividad, Bioquímica, metabolismo y evaluación.

\section{Abstract}

The teaching of science has developed in recent years an important work in the application of Information and Communication Technologies (ICT) in dassrooms. Given the above and the need to adapt the ICT to educational context and the developments in the field of teaching and chemistry, this artide reveals a proposal which integrates these elements combining the Biochemistry with an Interactive Didactic Unit, taking into account the approaches of Model of Problem Solving.

To implement the project we took into account the educational standards in Colombia and various documents related to curriculum standards associated to concepts of biochemistry, and as their historical and epistemological processes.

Keywords

Didactic Unit, virtuality, interactivity, Biochemistry, metabolism and evaluation. 


\section{Desarrollo}

La educación actual exige al docente y a las instituciones educativas crear herramientas didácticas y pedagógicas que vayan a la par con los constantes cambios científicos, teconológicos y sociales. Una de estas herramientas adoptadas en los últimos años es la informática, la aul ha tenido una considerable influencia en la actividad humana y junto con la internet ha establecido canales de comunicación cuyas características permiten mantener un interesante dinamismo de los procesos humanos y en particular los educativos (Pérez, 2003). Es así que el docente de hoy se ve en la necesidad de abordar en campos como la creación de softwares y paginas web, que le permitan no solo darle un nuevo matiz a su labor educativa sino además, consolidar un ambiente de aprendizaje dinámico y efectivo en el aula.

La educación en Colombia no se encuentra ajena a las transformaciones educativas que se han venido dando internacionalmente, por lo tanto ha planteado a través de entes como el Ministerio de Educación Nacional y la Secretaria de Educación Distrital de Bogotá D.C., estándares educativos (MEN, 2006) y orientaciones curriculares (SED Bogotá, 2007) dirigidos haća la excelencia y el fortalecimiento de las instituciones educativas del país. El crecimiento del nivel académico en Ciencias Naturales y el fomento del uso de las Tecnologías, son algunos de los pilares sobre los cuales se soportan los principales cambios educativos, y es en este aspecto donde se fundamenta este proyecto ya que busca integrar las Tecnologías de la Informática y la Comunicación (TIC), La Didáctica de las Ciencias y como campo disciplinar la Bioquímica, por medio de una Unidad Didáctica Interactiva para el Aprendizaje de Bioquímica (UDIAB) que logre formar un puente entre el maestro y el estudiante de tal forma que el proceso de enseñanza y aprendizaje adquiera un carácter motivador, dinámico, amigable y novedoso.

El proyecto UDIAB considera que las TIC aplicadas en la educación deben poseer un contenido estructurado a partir de principios didácticos, es decir, las TIC deben ser una herramienta educativa diseñada teniendo en cuenta la manera en cómo el sujeto es capaz de aprender (Pérez, 2003).

La unidad didáctica interactiva pretende facilitar el aprendizaje de la Bioquímica partiendo de la premisa que todo conocimiento debe ser significativo para quien lo aprende. Es así que se proyecta que los estudiantes se familiaricen con los procesos químicos que se llevan a cabo dentro de su organismo permitiendo relacionar las ciencias naturales con su vida cotidiana, es decir, el objetivo principal de la unidad es lograr que el estudiante comprenda la Bioquímica partiendo de su entomo y lo que él conoce (ideas previas). El aprendizaje significativo (Ausubel, 1991) hace parte de la columna vertebral de este proyecto ya que involuca activamente al estudiante y su contexto social, esto permite proponer una investigación entorno a la UDIAB ya que es posible observar y controlar ciertas variables con las cuales se logre planificar y establecer una Unidad Didáctica de alto nivel para el proceso de enseñanza y aprendizaje de la Bioquímica. La vía para lograr el aprendizaje significativo a través de la UDIAB es la Resolución de Problemas, una de las estrategias más importantes en lo que se refiere a la Didáctica de las Ciencias Experimentales (Narváez, 1994).

La UDIAB esta pensada como una herramienta complementaria para los procesos de enseñanza-aprendizaje del área de Bioquímica y enfocada a los sistemas metabólicos (catabólicos y anabólicos) de la célula animal. De esta manera se busca que el estudiante se motive hacía el aprendizaje de las ciencias biológicas y químicas a partir de la interacción con las tecnologías informáticas y la Bioquímica del metabolismo. 


\section{Un planteamiento de evaluación basado en el uso de TIC.}

La Química está formada por un conjunto de conocimientos que se encuentran en evolución, debido a la continua valoración, revisión y análisis reflexivo de los fenómenos, por ende para realizar una formación en Química la evaluación debe ser un aspecto primordial. El término de evaluación ha tomado recientemente una nueva visión desde la perspectiva de la Didáctica y la Pedagogía, así la tesis principal que plantean estas ciencias sobre el significado real del concepto de evaluación, se basa en considerarla un proceso continuo y dinámico donde el real objetivo es fortalecer, retroalimentar y analizar el aprendizaje. Asimismo la evaluación contribuye a mejorar la enseñanza a partir del análisis de la incidencia de la práctica docente en los estudiantes y permite ajustar el currículo de acuerdo a las necesidades evidenciadas (Gil, 2005).

Teniendo en auenta lo anterior y en contraste con lo evidenciado en la etapa de diseño y aplicación de la UDIAB se ha logrado identificar aspectos relevantes de la evaluación como los que se presentan a continuación:

$>$ La evaluación debe ser un proceso continuo a lo largo de la aplicación de las TIC, es así que en la UDIAB se induye instrumentos como el KPSI y el Q-sort los cuales ofrecen esta posibilidad.

> Con la ayuda de las herramientas de evaluación que posee la UDIAB, el estudiante puede realizar un mejor proceso de autorregulación de sus aprendizajes.

$>$ Las TIC deben contar con la posibilidad de evaluar al estudiante de forma cualitativa y arantitativa.

\section{Bibliografía}

Ausubel, D. Novak J. (1991). Psicología Educativa: Un Punto de Vista Cognoscitivo, 2da Edición. México: Editorial Trillas.

Gil, Daniel y Martínez, Joaquín. ( ）. ¿Cómo promover el interés por la cultura científica? Una propuesta didáctica fundamentada para la educación cient́fica de jóvenes de 15 a 18 años. UNESCO. Santiago de Chile, Chile, 2005. P 159-182.

Ministerio de Educación Nacional. (2006) Estándares Básicos de Competencias en Lenguaje, Matemáticas, Ciencias y Ciudadanas. (1ra Edición) Bogotá D.C., Colombia: Ministerio de Educación Nacional.

Narvaez L y Duran L. (1994). Un Enfoque Constructivista en el Aprendizaje Significativo de Algunos Conceptos de Química Correspondientes al Programa de Ciencias naturales de Algunos Alumnos de Grado Sexto de Básica Secundaria. Tesis de Especialización en Docencia de la Biología no Publicada. Universidad Surcolombiana. Universidad del Tolima. Neiva, Colombia.

Pérez M. (2003). Nuevas Tecnologías y Educación. Grupo Docente. Revista On Line de Educación, 5. Recuperado el 22 de Enero de 2009 de la base de datos Océano Saber.

Secretaria de Educación de Bogotá D.C. (2007). Colegios Públicos de Excelencia para Bogotá: Orientaciones Curriculares para el Campo de Ciencia y Tecnología. Bogotá D.C., Colombia: Secretaria de Educación de Bogotá D.C. 